\title{
Takotsubo syndrome or myocardial stunning due to spasm of a non-obstructive left anterior descending coronary artery?
}

To the Editor:

Sabra et al reported on a 48-year-old woman with "atypical" takotsubo syndrome (TTS), associated with chest pain lasting for $\sim 60$ minutes, and with myocardial perfusion scintigraphy "showing reduced to absent perfusion in the anterior and anteroseptal segments", "gated images showing reduced anterior wall thickening", "left ventriculography showing anterior wall motion abnormality", coronary angiography revealing $50 \%$ stenosis of the proximal left anterior descending artery (LAD) and the first diagonal branch with a fractional flow reserve of 0.84 in both vessels", "left ventriculography showing anterolateral akinesia", echocardiography 48 hours after presentation showing improved contraction pattern with minor hypokinesis of the anteroseptal wall only", "cardiac magnetic resonance imaging showing a small area of edema in the anterior wall without signs of late gadolinium enhancement', and I-123 MIBG imaging suggesting of cardiac denervation. ${ }^{1}$ The authors contrasted their report with an earlier one of a 71-year-old woman with TTS, who showed a normal perfusion during the acute phase. ${ }^{2}$ However that patient underwent myocardial perfusion imaging earlier, and immediately after experiencing chest pain in the setting of an exercise stress test, and one wonders whether at the onset of TTS myocardial perfusion is normal, and it becomes abnormal $\geq 1$ hour(s) later due to ensuing post-ischemic myocardial stunning leading to myocardial edema, compressing the microcirculation externally. ${ }^{3}$ Indeed the authors of the other report, ${ }^{2}$ alluded to abnormal myocardial perfusion a few hours after the onset of TTS. Something that is "atypical" about the present case, ${ }^{1}$ is that it showed left ventricular wall motion abnormalities (LVWMA) in the septal and anterior left ventricular territories, matching the area of decreased myocardial perfusion, and corresponding to a possible transient curtailment of coronary flow to the LAD, due to, for example coronary vasospasm, while TTS is characterized by LVWMA of circumferential distribution, beyond the territorial distribution of a single coronary artery.

John E. Madias, MD, FACC, FAHA, Icahn School of Medicine at Mount Sinail Cardiology Division, Elmhurst Hospital Center, 79-01 Broadway, Elmhurst, NY 11373,USA; madiasj@nychhc.org.

\section{Disclosures}

None.

\section{References}

1. Sabra MMM, Costa FS, de Azevedo JC, Mesquita CT, Verberne HJ. Myocardial perfusion scintigraphy during chest pain: an atypical presentation of takotsubocardiomyopathy? J Nucl Cardiol. 2018. https://doi.org/10.1007/s12350-018-1286-8.

2. Dorfman T, Aqel R, Allred J, Woodham R, Iskandrian AE. Takotsubo cardiomyopathy induced by treadmill exercise testing: an insight into the pathophysiology of transient left ventricular apical (or midventricular) ballooning in the absence of obstructive coronary artery disease. J Am Coll Cardiol. 2007;49:1223-5.

3. Madias JE. Coronary vasospasm is an unlikely cause of Takotsubo syndrome, although we should keep an open mind. Int J Cardiol. 2014;176:1-5.

doi:10.1007/s12350-018-1347-z

Funding No funding was received for this work.

J Nucl Cardiol 2020;27:1053.

$1071-3581 / \$ 34.00$

Copyright (c) 2018 American Society of Nuclear Cardiology. 